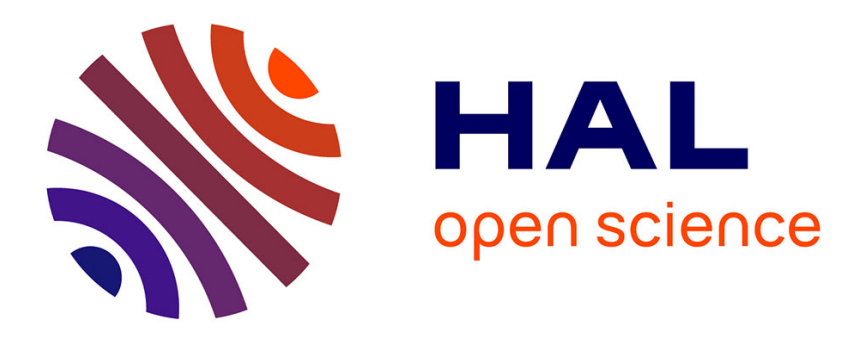

\title{
Boundary effects on the maximal angle of stability of a granular packing
}

Philippe Boltenhagen

\section{To cite this version:}

Philippe Boltenhagen. Boundary effects on the maximal angle of stability of a granular packing. Physical Review E , 1999, 12 (1), pp.135 - 142. 10.1007/s100510050979 . hal-02380144

HAL Id: hal-02380144

https://hal.science/hal-02380144

Submitted on 26 Nov 2019

HAL is a multi-disciplinary open access archive for the deposit and dissemination of scientific research documents, whether they are published or not. The documents may come from teaching and research institutions in France or abroad, or from public or private research centers.
L'archive ouverte pluridisciplinaire HAL, est destinée au dépôt et à la diffusion de documents scientifiques de niveau recherche, publiés ou non, émanant des établissements d'enseignement et de recherche français ou étrangers, des laboratoires publics ou privés. 


\title{
Boundary effects on the maximal angle of stability of a granular packing
}

\author{
P. Boltenhagen ${ }^{\mathrm{a}}$ \\ Laboratoire de Dynamique des Fluides Complexes ${ }^{\text {b }}, 3$ rue de l'Université, 67084 Strasbourg Cedex, France
}

Received 1 April 1999

\begin{abstract}
We have studied the maximal angle of stability of a granular packing confined between two walls. The effect of the walls is to increase the angle dramatically. The decay of the angle with the distance between the walls is exponential with a characteristic length which is a function of the beads diameter. The effect of the roughness of the walls has been also studied.

PACS. 81.05.Rm Porous materials; granular materials - 83.70.Fn Granular solids
\end{abstract}

\section{Introduction}

Physics of granular matter has been the subject of various works in the recent years. This infatuation comes from the fact that although granular matter is very familiar for everybody its behaviour is extremely interesting and unexpected: as an example, the stress distribution at the bottom of a heap of sand does exhibit a minimum straight below the apex of the sandpile reflecting the presence of lines of stress [1].

The behaviour of granular materials is also of a great industrial interest as for example the grinding, the mixing and the storage. Many physicists have tried to understand the basic comportment of such systems and we can cite for example the interesting problem of silos [2-5].

An other interesting point is that granular matter behaves like a liquid as it flows and conforms the shape of a container, but exhibits a solid like behaviour as it can sustain under gravity a finite angle of repose $\theta_{\mathrm{r}}$. This angle is defined at rest as follow: for slopes such as $\theta<\theta_{\mathrm{r}}$ no flow of sand can occur and the sandpile behaves like a solid whereas for $\theta>\theta_{\mathrm{r}}$ the top layers of sand flow freely. This angle will be the one of a heap of sand created by pouring the material from a single source point onto an infinite plate. Many experimental measurements [6,7] have shown that $\theta_{\mathrm{r}}$ is highly dependent on the shape and surface roughness of the grains as it varies typically from $22^{\circ}$ for smooth spherical grains up to $60^{\circ}$ for angular and rough grains. The angle of repose can also be modified in the presence of walls as studied by Grasselli and Hermann [8].

As noticed by Bagnolds [9], an other interesting angle observed in the physics of granular matter is the angle of maximal stability $\theta_{\mathrm{m}}$. This angle, several degrees larger than $\theta_{\mathrm{r}}$, is the maximal angle that a granular medium can

\footnotetext{
${ }^{a}$ e-mail: bolten@ldfc.u-strasbg.fr

b UMR 7506 CNRS
}

reach when carefully tilted. Above $\theta_{\mathrm{m}}$ grains start to flow and a macroscopic avalanche of grains is observed at the surface of the packing. After the avalanche the angle of the pile is $\theta_{\mathrm{r}}$.

Cohesion forces play also an important role in the physics of granular matter as it can change greatly the values of $\theta_{\mathrm{r}}$ and $\theta_{\mathrm{m}}[10]$.

In this paper we were interested by the study of the dependence of the maximal angle of stability on the presence of walls. Careful experiments and measurements of $\theta_{\mathrm{m}}$ reveal an important role played by the walls. The presence of boundaries increases greatly the maximal angle of stability reflecting the effect of the force lines that are supported by the walls making arches and leading to a more stable structure.

\section{Experimental set-up}

Experiments have been performed using a rectangular Plexiglas cell with a variable width $d$. The length of the cell is $25.5 \mathrm{~cm}$ and the height is $7.8 \mathrm{~cm}$ ( $c f$. Fig. 1). The width of the cell has been varied from $2 \mathrm{~mm}$ to $89 \mathrm{~mm}$. The cell is placed onto a tilting device that enables to determine the maximal angle of stability at which an avalanche is observed. During this work we have used monodisperse glass beads assembly with a diameter $D$ of 1,2 and $4 \mathrm{~mm}$. In order to get a relatively high precision of the measured values of $\theta_{\mathrm{m}}$, each experiment has been repeated from ten to thirty times in order to get an average value with a sufficiently small error bar. For each point the cell is filled with a new fabric up to the top.

As our beads are relatively large, humidity effects of the room and electrostatic effects arising from the friction when filling the cell can be neglected. 


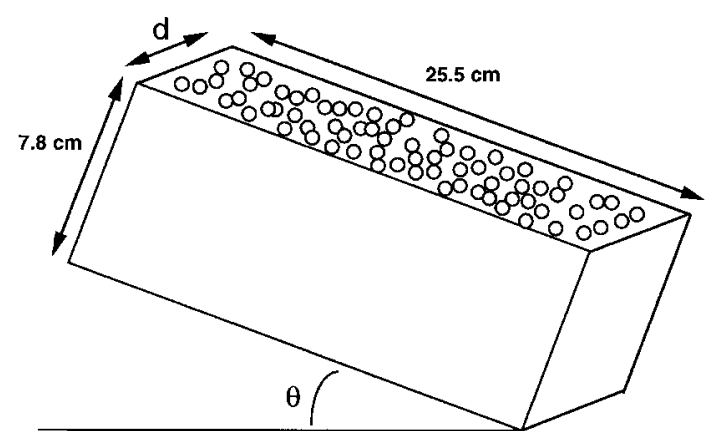

Fig. 1. Experimental set-up. The width $d$ of the cell is variable.

\section{Experimental results}

\subsection{Effect of the walls on the maximal angle of stability}

Before going through the results that we have obtained, it seems to be clear that the maximal angle of stability of the material packing will be highly dependent on the presence of boundaries. This arises from the fact that when filling and tilting the cell, beads form bridges between the walls that stabilise the structure. A priori we can emphasize that the presence of walls will increase $\theta_{\mathrm{m}}$.

In order to get the variation of $\theta_{\mathrm{m}}$ as a function of the distance between walls we have measured the angle at which a macroscopic avalanches is observed. By macroscopic we understand an avalanche where a great number of beads are involved. Events where only a very few beads are involved are not taken into account as they are by nature different from the others. We define the parameter $\lambda$ as the ratio between the distance between the walls over the diameter of the beads $(\lambda=d / D)$.

We present in Figure 2 the variation of the angle $\theta_{\mathrm{m}}$ as a function of $\lambda$ for the beads with a diameter of $4 \mathrm{~mm}$. The maximal angle of stability ranges from $46^{\circ}$ when $\lambda=1.5$ to $31.3^{\circ}$ when $\lambda=22.5$. When $\lambda$ becomes larger than about 9 the effect of the walls becomes absolutely negligible with the accuracy of our experiment. For $\lambda$ smaller than 9 the effect of the walls is clearly pointed out: the presence of walls increases the maximal angle of stability. The effect is the same for the beads with a diameter of 1 and $2 \mathrm{~mm}$, only the value of $\lambda$ where the effect of the walls is felt changes, this is shown in the next section.

The behaviour of the maximal angle of stability as a function of $\lambda$ can be examined by plotting the normalised ratio $\tau=\left[\theta(d)-\theta_{\infty}\right] / \theta_{\infty}$ as a function of $\lambda$ where $\theta_{\infty}$ is the maximal angle of stability where the walls play no role [8]. In our case $\theta_{\infty} \approx 31.3^{\circ}$. In the insert of Figure 2 we have plotted $\tau$ as a function of $\lambda$ in a semi-log plot for data with an accuracy high enough to eliminate the noise of the large $\lambda$ tail. We found that the behaviour of the maximal angle of stability as a function of $\lambda$ is an exponential law of the form $\theta(d)=\theta_{\infty}\left[1-\exp \left(-\lambda / \lambda^{*}\right)\right]$, where $\lambda^{*}$ is a dimensionless length over which most of the effect of the wall vanishes. In our case we found $\lambda^{*} \approx 2.53$ for the beads with a diameter of $4 \mathrm{~mm}$.

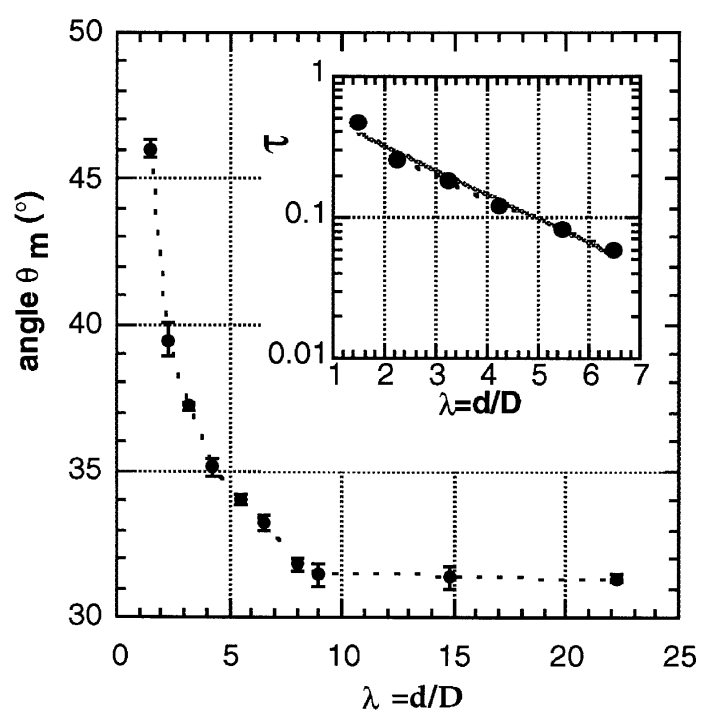

Fig. 2. Variation of the maximal angle of stability of a granular packing as a function of the parameter $\lambda=d / D$, ratio of the width of the cell over the diameter of the beads. The walls act to increase the stability of the packing by bridges formation. The dimensionless parameter $\tau=\left[\theta(d)-\theta_{\infty}\right] / \theta_{\infty}$ is plotted as a function of $\lambda$ and exhibits an exponential decay.

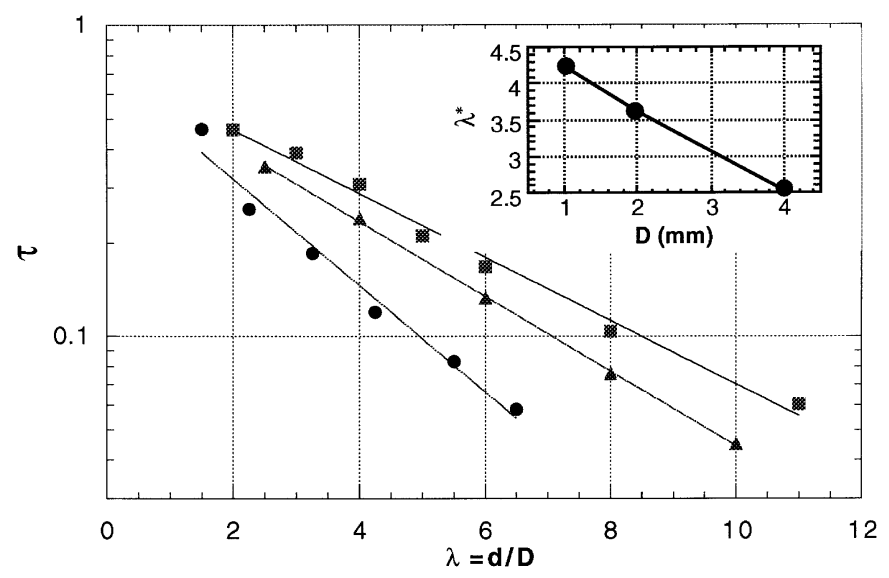

Fig. 3. Semi-log plot of the dimensionless parameter $\tau=$ $\left[\theta(d)-\theta_{\infty}\right] / \theta_{\infty}$ as a function of $\lambda$ for various beads diameter (circles $4 \mathrm{~mm}$, triangles $2 \mathrm{~mm}$, squares $1 \mathrm{~mm}$ ). Each curve is fitted by an exponential law with a characteristic length $\lambda^{*}$ over which most of the effect of the walls vanishes. The characteristic length $\lambda^{*}$ is a decreasing function of the beads diameter as shown in the insert.

\subsection{Effect of the diameter of the beads on the penetration length $\lambda^{*}$}

The effect of the beads diameter on the dimensionless length $\lambda^{*}$ has also been studied. For this purpose spherical glass beads with a diameter of $1 \mathrm{~mm}$ and $2 \mathrm{~mm}$ have been used. The procedure for measuring the maximal angle of stability of the granular packing is the same as for the $4 \mathrm{~mm}$ glass beads. We show in Figure 3 the results that we have obtained. The normalised ratio $\tau=\left[\theta(d)-\theta_{\infty}\right] / \theta_{\infty}$ is plotted for the three families of beads as a function 


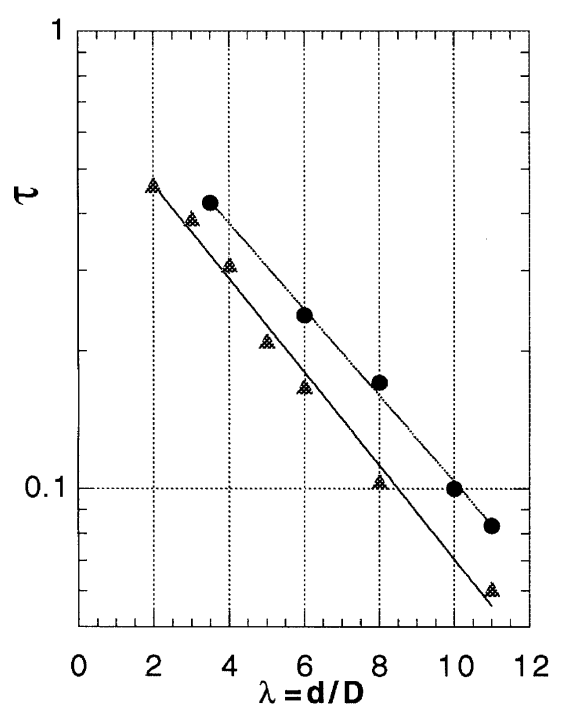

Fig. 4. Effect of the roughness of the walls (triangles, smooth surfaces-circles, rough surfaces). The roughness of the walls acts to increase the maximal angle of stability and the characteristic length $\lambda^{*}$.

of $\lambda$. The behaviour is governed by an exponential law with a characteristic length $\lambda^{*}$ that depends on the beads diameter $D$. Namely the characteristic length $\lambda^{*}$ is a decreasing function of $D$. We show in the insert of Figure 3 the behaviour of $\lambda^{*}$ as a function of $D$, we obtained an almost linear relation between $\lambda^{*}$ and $D$. The fact that $\lambda^{*}$ decreases with $D$ is not the behaviour that is expected, nevertheless we may propose the following explanation. The effect of the walls on the maximal angle of stability can be fully explained by the presence of bridges. Each bridge is hanged to the walls through frictional forces. The beginning of an avalanche is when some bridges break or slip along the walls. This will be easier if the weight carried by a bridge is large. Hence the breaking or the slipping will preferred for heavy beads, this may explain the observed behaviour. This is also consistent with the fact that for a given $\lambda, \tau$ is larger for small beads. Moreover the number of contact beads-wall is a decreasing function of the beads diameter, hence the critical stress necessary to induce an avalanche will be a decreasing function of $D$.

\subsection{Effect of the roughness of the walls}

The presence of the walls increases greatly the maximal angle of stability of the granular packing. This is due to the fact that grains form bridges between the walls that stabilise the structure. Bridges are hanged to the walls by frictional forces. By changing the contact between the walls and the beads we may try to see an effect. This has been verified by the following procedure: we have glued on the inner surfaces of the cell sand paper with an average roughness of 120 microns. The presence of the sand paper is to increase the frictional forces. We have used beads with a diameter of $1 \mathrm{~mm}$ and measured the maximal angle of stability of the granular packing. The results that we have obtained are shown in Figure 4. The effect of the sand paper is double: first one observe that the value of $\tau=\left[\theta(d)-\theta_{\infty}\right] / \theta_{\infty}$ is larger for a given value of $\lambda$ in the presence of the sand paper. This means that the roughness of the surface increases the maximal angle of stability for a given $\lambda$. This confirm the fact that the stability of the packing is effectively driven by the presence of bridges: the more the friction between the walls and the beads is large the more stable is the structure. Secondly the value of the dimensionless length $\lambda^{*}$ increases slightly in presence of sand paper. We obtained $\lambda^{*}=4.25$ for smooth surfaces and $\lambda^{*}=4.60$ for rough surfaces.

\section{Conclusion}

As a conclusion to this work we have demonstrated that the presence of walls affects greatly the maximal angle of stability of a granular packing. The stability of the packing comes certainly from arches that stabilise the structure. The decay of the maximal angle of stability as a function of the distance between the walls is exponential, with a characteristic length that decreases with the beads diameter. An interesting following of this work will be to study the complete dependence of $\lambda^{*}$ with $D$, with a more complete set of beads, as the linear relation should certainly vanish for very small beads and large one. By changing the roughness of the walls we may increase the stability of the structure.

\section{References}

1. J. Smid, J. Novosad, in Proceedings of the 1981 Powtech Conference, Ind. Chem. Eng. Symp. 63, D3v, 1-12 (1981).

2. S.F. Edwards, R.B. Oakeshott, Physica Amsterdam D 38, 88-93 (1989).

3. J.P Bouchaud, M.E. Cates, P. Claudin, J. Phys. I France 5, 639 (1995).

4. R.L. Brown, J.C. Richard, Principles of Powder Mechanics (Pergamon, New York, 1966).

5. P. Claudin, J.P. Bouchaud, Phys. Rev. Lett. 78, 231 (1997).

6. H.M. Jaeger, C.H. Liu, S.R. Nagel, Phys. Rev. Lett. 62, 40 (1988).

7. P. Evesque, D. Fargeix, P. Habib, M.P. Luong, P. Porion, Phys. Rev. E 47, 2326 (1993).

8. Y. Grasselli, H.J. Hermann, Physica A 246, 301-312 (1997).

9. R.A. Bagnolds, Proc. Roy. Soc. A 225, 49-63 (1954); 2326 (1993).

10. R. Albert, I. Albert, D. Hornbaker, P. Schiffer, A-L Barabasi, Phys. Rev. E 56, R6271 (1997). 